\title{
Could we have predicted the recent downturn in the South African housing market?
}

\author{
Sonali Das ${ }^{\mathrm{a}, 1}$, Rangan Gupta ${ }^{\mathrm{b}, *}$, Alain Kabundi ${ }^{\mathrm{c}, 2}$ \\ ${ }^{a}$ Logistics and Quantitative Methods, CSIR Built Environment, P.O. Box 395, Pretoria 0001, South Africa \\ ${ }^{\mathrm{b}}$ Department of Economics, University of Pretoria, Pretoria 0002, South Africa \\ ${ }^{\mathrm{c}}$ University of Johannesburg, Department of Economics and Econometrics, Johannesburg 2006, South Africa
}

\section{A R T I C L E I N F O}

\section{Article history:}

Received 14 October 2008

Available online $\mathrm{xxxx}$

JEL classification:
C11
C13
C33
C53
Keywords:
Dynamic factor model
BVAR
Forecast accuracy

Forecast accuracy

\begin{abstract}
A B S T R A C T
This paper develops large-scale Bayesian Vector Autoregressive (BVAR) models, based on 268 quarterly series, for forecasting annualized real house price growth rates for large-, medium- and small-middle-segment housing for the South African economy. Given the in-sample period of 1980:01-2000:04, the large-scale BVARs, estimated under alternative hyperparameter values specifying the priors, are used to forecast real house price growth rates over a 24-quarter out-of-sample horizon of 2001:01-2006:04. The forecast performance of the large-scale BVARs are then compared with classical and Bayesian versions of univariate and multivariate Vector Autoregressive (VAR) models, merely comprising of the real growth rates of the large-, medium- and small-middle-segment houses, and a large-scale Dynamic Factor Model (DFM), which comprises of the same 268 variables included in the large-scale BVARs. Based on the one- to four-quarters-ahead Root Mean Square Errors (RMSEs) over the out-of-sample horizon, we find the large-scale BVARs to not only outperform all the other alternative models, but to also predict the recent downturn in the real house price growth rates for the three categories of the middle-segmenthousing over the period of 2003:01-2008:02.
\end{abstract}

(c) 2009 Elsevier Inc. All rights reserved.

\section{Introduction}

This paper develops large-scale Bayesian Vector Autoregressive (BVAR) models, based on 268 quarterly series, for forecasting annualized real house price growth rates, where real house price is the ratio of the nominal house price to the Consumer Price Index (CPI), for large-, medium- and small-middle-segment housing for the South

\footnotetext{
* Corresponding author. Fax: +27 123625207.

E-mail addresses: SDas@csir.co.za (S. Das), Rangan.Gupta@up.ac.za (R. Gupta), akabundi@uj.ac.za (A. Kabundi).

1 Fax: +27 128413037

${ }^{2}$ Fax: +27 115593039 .
}

African economy. ${ }^{3}$ Given the in-sample period of 1980:01-2000:04, the large-scale BVARs, estimated under alternative hyperparameter values specifying the priors, are used to forecast real house price growth rates over a

\footnotetext{
${ }^{3}$ Data on house prices are obtained from the ABSA Housing Price Survey, with ABSA being one of the leading private banks of South Africa. The ABSA Housing Price Survey, distinguishes between three price categories as luxury houses (R2.6-R9.5 million), middle-segment houses (R226,000-R2.6 million) and affordable houses (R226,000 and below with an area in the range of $40-79 \mathrm{~m}^{2}$ ); and further subdivides the middle segment category based on the square meters of house area into small $\left(80-140 \mathrm{~m}^{2}\right)$, medium $\left(141-220 \mathrm{~m}^{2}\right)$ and large $\left(221-400 \mathrm{~m}^{2}\right)$. Given the easy accessibility of house price data for the middle-segment houses, we restrict our study to this category only. However, given that the market for different house-sizes within this category, behave differently (Burger and van Rensburg, 2008), we consider each of them separately, rather than investigating the overall house price of the middle-segment of the South African housing market.
} 


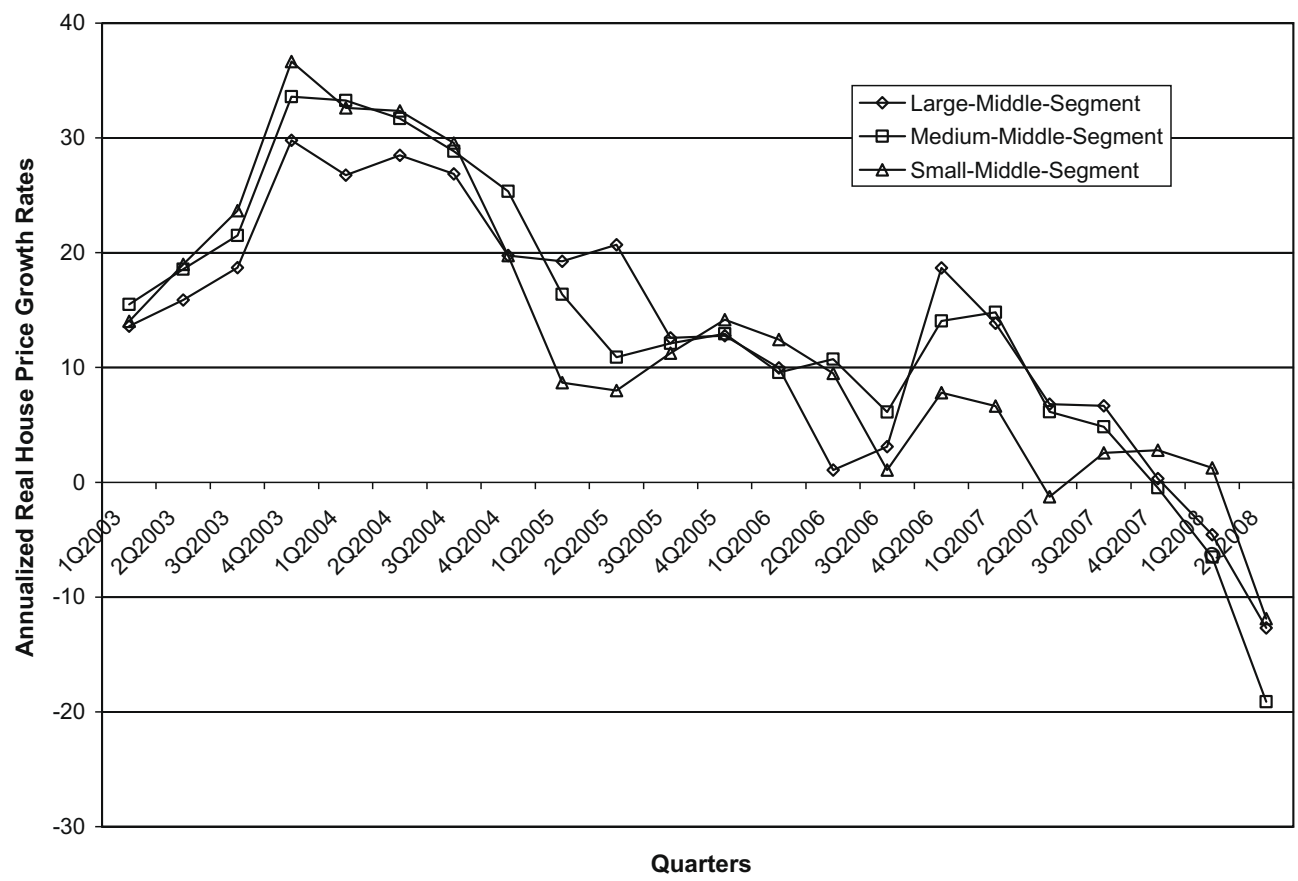

Fig. 1. Recent downturn in the middle-segment housing market (2003:01-2008:02).

24-quarter out-of-sample horizon of 2001:01-2006:04. ${ }^{4}$ The forecast performance of the large-scale BVARs are then compared with classical and Bayesian versions of univariate and multivariate Vector Autoregressive (VAR) models, merely comprising of the real growth rates of the large-, mediumand small-middle-segment houses, and a large-scale Dynamic Factor Model (DFM), which comprises of the same 268 variables included in the large-scale BVARs. Once we determine a model that produces, on average, the minimum one- to four-quarters-ahead Root Mean Square Errors (RMSEs) over the out-of-sample horizon, we evaluate, how well the same would have been able to predict the recent downturn, depicted in Fig. 1, in the real house price growth rates for the three categories of the middle-segment-housing over the period of 2003:01-2008:02.

At this stage, two questions arise: First, why is forecasting real house price growth rates important? And second, why develop large-scale BVARs for this purpose? As far as the answer to the first question is concerned, the importance of predicting house price inflation is motivated by recent studies that conclude that asset prices help forecast both inflation and output (Forni et al., 2003; Stock and Watson, 2003; Gupta and Das, 2008 and Das et al., 2008). Since a large amount of individual wealth is imbedded in houses, similar to other asset prices, house price movements are thus important in signaling inflation. ${ }^{5}$ As such, models that

\footnotetext{
${ }^{4}$ The choice of the out-of-sample period is exactly the same as used by two recent studies on the South African housing market, namely, Gupta and Das (2008) and Das et al. (2008).

${ }^{5}$ Gupta and Das (2008) point out that in South Africa, housing inflation and CPI inflation tend to move together, though the former, understandably, is more volatile.
}

forecast real house price inflation can give policy makers an idea about the direction of overall inflation in the future, and hence, can provide a better control for designing of appropriate policies. Ideally, one would want to forecast key macroeconomic variables based on models that have strong theoretical structures, such as Dynamic Stochastic General Equilibrium Models which are immune to the Lucas (1976) critique. However, the usage of a atheoretical framework like that of the VAR allows one to provide a preliminary and quick analysis of where the economy is headed. The rationale for large-scale BVARs to forecast real house price growth rates emanates from the fact that a large number of economic variables help in predicting real housing price growth (Cho, 1996; Abraham and Hendershott, 1996; Johnes and Hyclak, 1999; Rapach and Strauss, 2007, 2009). For instance, income, interest rates, construction costs, labor market variables, stock prices, industrial production, consumer confidence index, and also variables containing information about the world economy and the major trading partners of South Afri$\mathrm{ca}$ - which are included in the large-scale BVARs, are potential predictors. In addition, given that movements in the housing market are likely to play an important role in the business cycle, not only because housing investment is a very volatile component of demand (Bernanke and Gertler, 1995), but also because changes in house prices tends to have important wealth effects on consumption (International Monetary Fund, 2000) and investment (Topel and Rosen, 1988), the importance of forecasting house price inflation is vital. The housing sector thus plays a significant role in acting as leading indicator of the real sector of the economy, and as such, predicting it correctly cannot be overemphasized, especially in the light of the recent credit crunch in the U.S. that started with the burst of the housing price bubble which, in 
turn, transmitted to the real sector of the economy driving it towards an imminent recession. Besides this, the fact that BVARs are quite well-suited in predicting turning points of macroeconomic variables have recently been substantiated by Dua and Ray (1995), Del Negro (2001), Gupta and Sichei (2006), Gupta (2006, 2009), Banerji et al. (2008), Zita and Gupta (2008) and Gupta and Das (forthcoming) amongst others.

To realize the contribution of this study, it is important to place this paper in the context of current research that has been done on forecasting the housing market. In this regard, few studies that are worth mentioning are: Rapach and Strauss (2007, 2009), Gupta and Das (2008, forthcoming), Das et al. (2008). Rapach and Strauss (2007) used an autoregressive distributed lag (ARDL) model framework, containing 25 determinants, to forecast real housing price growth for the individual states of the Federal Reserve's Eighth District. Given the difficulty in determining apriori the particular variables that are most important for forecasting real housing price growth, the authors also use various methods to combine the individual ARDL model forecasts, which result in better forecast of real housing price growth. Rapach and Strauss (2009) look at doing the same for 20 largest US states based on ARDL models containing large number of potential predictors, including state, regional and national level variables. Once again, the authors reach similar conclusions as far as the importance of combining forecasts are concerned. Given that in practice, forecasters and policymakers often extract information from many series than the ones included in smaller models, like the ones used by Rapach and Strauss (2007, 2009), who also indicate the importance of combining forecast from alternative models, the role of a large-scale models cannot be ignored. In addition, one cannot condone the fact that the main problem of small models, as seen from the studies by Rapach and Strauss (2007, 2009), is in the decision regarding the choice of the correct potential predictors to be included. Due to this reason, Vargas-Silva (2008) uses a Factor Augmented VAR (FAVAR) model containing 120 monthly series to analyze the impact of monetary policy actions on the housing sector of four different regions of the United States. Further, Das et al. (2008) show that forecast performances of Spatial BVARs (SBVARs), developed by Gupta and Das (2008), to predict regional house prices in the middle-segment housing category of South Africa, can be markedly improved using a DFM. Clearly then, the motivation and the need to forecast house prices using large-scale models is quite compelling. However, to the best of our knowledge, this is the first attempt to look into the ability of large-scale BVARs in forecasting and predicting downturns in real house price growth rates. ${ }^{6,7}$ The only other study that does look into

\footnotetext{
${ }^{6}$ Note, Dua and Smyth (1995), Dua and Miller (1996) and Dua et al. (1999) used coincident and leading indexes in BVAR models to forecast home sales for the Connecticut and the overall US economy, respectively.

7 Note, even though just like Gupta and Das (2008) and Das et al. (2008), we look at the middle-segment of the South African housing market, unlike them, we do not look into regional data. This is simply because the recent downturn in the housing market has been an economy-wide phenomenon and not been restricted to any specific region (ABSA Housing Price Review (2008)Q1, Q2 and Q3).
}

forecasting the recent downturn in real house price growth rates for the 20 largest states of the US economy, is Gupta and Das (forthcoming). In this paper, the authors use SBVARs, based merely on real house price growth rates, to predict their downturn over the period of 2004:012008:01. They find that, though the models are quite wellequipped in predicting the recent downturn, they underestimate the decline in the real house price growth rates by quite a margin. They attribute this underprediction of the models to the lack of any information on fundamentals in the estimation process. Against this backdrop, our paper can thus be viewed as an extension of the abovementioned studies, in the sense that we not only use large-scale BVARs that allow for the role of a widest possible set of domestic, foreign and world fundamentals to affect the housing sector, but also use them to predict the recent downturn in the South African housing market. At this juncture, we must elaborate that given the type of models that we use, the study can easily be carried out for any country(ies). The reason behind using the South African housing market as a case study is simply data driven, especially, as far as data on the 268 macroeconomic variables are concerned, which, in turn, were obtained from the recent study of Gupta and Kabundi (2008a,b,c) and Das et al. (2008). ${ }^{8}$ The remainder of the paper is organized as follows: Section 2 lays out the basics of the benchmark large-scale BVARs and the alternative models, while, Section 3 discusses the data. Sections 4 and 5, respectively, evaluate the forecasting performances of the models and their ability to predict the recent downturn in the housing market. Finally, Section 6 concludes.

\section{The model}

\subsection{VARs and BVARs}

The Vector Autoregressive (VAR) model, though 'atheoretical', is particularly useful for forecasting purposes. An unrestricted VAR model, as suggested by Sims (1980), can be written as follows:

$y_{t}=A_{0}+A(L) y_{t}+\varepsilon_{t}$

where $y$ is a $(n \times 1)$ vector of variables being forecasted; $A(L)$ is a $(n \times n)$ polynomial matrix in the backshift operator $L$ with lag length $p$, i.e., $A(L)=A_{1} L+A_{2} L^{2}+\cdots+A_{p} L^{p}$; $A_{0}$ is a $(n \times 1)$ vector of constant terms, and $\varepsilon$ is a $(n \times 1)$ vector of error terms. In our case, we assume that $\varepsilon \sim N\left(0, \sigma^{2} I_{n}\right)$, where $I_{n}$ is a $n \times n$ identity matrix.

Note the VAR model, generally uses equal lag length for all the variables of the model. One drawback of VAR models is that many parameters need to be estimated, some of which may be insignificant. This problem of overparameterization, resulting in multicollinearity and a loss of degrees of freedom, leads to inefficient estimates and possibly large out-of-sample forecasting errors. One solution, often adapted, is simply to exclude the insignificant lags based on statistical tests. Another approach is to use a near VAR, which specifies an unequal number of lags for the different equations.

\footnotetext{
${ }^{8}$ See Section 3 for further details.
} 
However, an alternative approach to overcoming this overparameterization, as described in Litterman (1981, 1986), Doan et al. (1984), Todd (1984), and Spencer (1993), is to use a BVAR model. Instead of eliminating longer lags, the Bayesian method imposes restrictions on these coefficients by assuming that they are more likely to be near zero than the coefficients on shorter lags. However, if there are strong effects from less important variables, the data can override this assumption. The restrictions are imposed by specifying normal prior distributions with zero means and small standard deviations for all coefficients with the standard deviation decreasing as the lags increase. The exception to this is that the coefficient on the first own lag of a variable has a mean of unity. Litterman (1981) used a diffuse prior for the constant. This is popularly referred to as the 'Minnesota prior' due to its development at the University of Minnesota and the Federal Reserve Bank at Minneapolis.

Formally, as discussed above, the means of the Minnesota prior take the following form:

$\beta_{i} \sim N\left(1, \sigma_{\beta_{i}}^{2}\right) \quad$ and $\beta_{j} \sim N\left(0, \sigma_{\beta_{j}}^{2}\right)$

where $\beta_{i}$ denotes the coefficients associated with the lagged dependent variables in each equation of the VAR, while $\beta_{j}$ represents any other coefficient. In the belief that lagged dependent variables are important explanatory variables, the prior means corresponding to them are set to unity. However, for all the other coefficients, $\beta_{j}$ 's, in a particular equation of the VAR, a prior mean of zero is assigned to suggest that these variables are less important to the model.

The prior variances $\sigma_{\beta_{i}}^{2}$ and $\sigma_{\beta_{\beta}}^{2}$, specify uncertainty about the prior means $\bar{\beta}_{i}=1$, and $\bar{\beta}_{j}=0$, respectively. Because of the overparameterization of the VAR, Doan et al. (1984) suggested a formula to generate standard deviations as a function of small numbers of hyperparameters: $w, d$, and a weighting matrix $f(i, j)$. This approach allows the forecaster to specify individual prior variances for a large number of coefficients based on only a few hyperparameters. The specification of the standard deviation of the distribution of the prior imposed on variable $j$ in equation $i$ at lag $m$, for all $i, j$ and $m$, defined as $\sigma_{i j m}$, can be specified as follows:

$\sigma_{i j m}=[w \times g(m) \times f(i, j)] \frac{\hat{\sigma}_{i}}{\hat{\sigma}_{j}}$

with $f(i, j)=1$, if $i=j$ and $k_{i j}$ otherwise, with $\left(0 \leqslant k_{i j} \leqslant 1\right)$, $g(m)=m^{-d}, d>0$. Note that $\hat{\sigma}_{i}$ is the estimated standard error of the univariate autoregression for variable $i$. The ratio $\hat{\sigma}_{i} / \hat{\sigma}_{j}$ scales the variables to account for differences in the units of measurement and hence causes specification of the prior without consideration of the magnitudes of the variables. The term $w$ indicates the overall tightness and is also the standard deviation on the first own lag, with the prior getting tighter as we reduce the value. The parameter $g(m)$ measures the tightness on lag $m$ with respect to lag 1 , and is assumed to have a harmonic shape with a decay factor of $d$, which tightens the prior on increasing lags. The parameter $f(i, j)$ represents the tightness of variable $j$ in equation $i$ relative to variable $i$, and by increasing the interaction, i.e., the value of $k_{i j}$, we can loosen the prior. ${ }^{9}$ Note, in the standard Minnesota-type prior, the overall tightness $(w)$ takes the values of $0.1,0.2$ and 0.3 , while, the lag decay $(d)$ is generally chosen to be equal to $0.5,1.0$ and 2.0 . The interaction parameter $\left(k_{i j}\right)$ is traditionally set at $=0.5$. The small-scale BVARs would be estimated with this set of parameterization of the priors.

Given that we have domestic as well as foreign and world variables in the 268 data series used for the largescale models, and realizing that South Africa is a small open economy, and hence, domestic variables would have minimal, if any, effect on foreign and world variables, while the latter set of variables is sure to have an influence on the South African variables, setting $k_{i j}=0.5$ could be a quite far fetched from reality. Hence, borrowing from the BVAR models used for regional forecasting, involving both regional and national variables, and following Kinal and Ratner (1986), Shoesmith (1992) and Gupta and Kabundi (2008b,c), the weight of a foreign or world variable in a foreign or world equation, as well as a domestic equation, is set at 0.6. The weight of a domestic variable in other domestic equation is fixed at 0.1 and that in a foreign or world equation at 0.01 . Finally, the weight of the domestic variable in its own equation is 1.0. These weights are in line with Litterman's circle-star structure. Star (foreign or world) variables affect both star and circle (domestic) variables, while circle variables primarily influence only other circle variables. ${ }^{10}$ Clearly then, the large-scale BVARs are estimated with asymmetric priors.

Finally, once the priors have been specified, the alternative BVARs, whether based on 1 or 3 or all the 268 variables, are estimated using Theil (1971) mixed estimation technique. Specifically, suppose we denote a single equation of the VAR model as: $y_{1}=X \beta+\varepsilon_{1}$, with $\operatorname{Var}\left(\varepsilon_{1}\right)=\sigma^{2} I$, then the stochastic prior restrictions for this single equation can be written as:

$\left[\begin{array}{c}M_{111} \\ M_{112} \\ \cdot \\ \cdot \\ \cdot \\ M_{n n p}\end{array}\right]=\left[\begin{array}{cccccc}\sigma / \sigma_{111} & 0 & . & . & . & 0 \\ 0 & \sigma / \sigma_{112} & 0 & . & . & 0 \\ \cdot & \cdot & . & . & . & \cdot \\ \cdot & . & . & . & . & . \\ 0 & . & . & . & . & 0 \\ 0 & 0 & . & . & 0 & \sigma / \sigma_{n n p}\end{array}\right]\left[\begin{array}{c}a_{111} \\ a_{112} \\ \cdot \\ \cdot \\ \cdot \\ a_{n n p}\end{array}\right]+\left[\begin{array}{c}u_{111} \\ u_{112} \\ \cdot \\ \cdot \\ \cdot \\ u_{n n p}\end{array}\right]$

(4)

Note, $\operatorname{Var}(u)=\sigma^{2} I$ and the prior means, $M_{i j m}$, and prior variance, $\sigma_{i j m}$, take the forms shown in (2) and (3). With (4) written as:

$r=R \beta+u$

and the estimates for a typical equation are derived as follows:

$\hat{\beta}=\left(X^{\prime} X+R^{\prime} R\right)^{-1}\left(X^{\prime} y_{1}+R^{\prime} r\right)$

\footnotetext{
${ }^{9}$ For an illustration, see Dua and Ray (1995).

10 We also experimented by assigning higher and lower interaction values, in comparison to those specified above, to the star variables in both the star and circle equations, but, the rank ordering of the alternative forecasts remained the same.
} 
Essentially then, the method involves supplementing the data with prior information on the distribution of the coefficients. The number of observations and degrees of freedom are increased by one in an artificial way, for each restriction imposed on the parameter estimates. The loss of degrees of freedom due to over-parameterization associated with a classical VAR model is, therefore, not a concern in the BVARs.

\section{2. $D F M$}

This study uses the Dynamic Factor Model (DFM) developed by Forni et al. (2005) to extract common components between macroeconomics series, and then these common components are used to forecast real house price growth rates in South Africa. In the VAR models, since all variables are used in forecasting, the number of parameters to be estimated depends on the number of variables $n$. With such a large information set, $n$, the estimation of a large number of parameters leads to a curse of dimensionality, especially in the case of classical VARs. The DFM uses information set accounted by few factors $q \ll n$, which transforms the curse of dimensionality into a blessing of dimensionality.

The DFM expresses individual times series as the sum of two unobserved components: a common component driven by a small number of common factors and an idiosyncratic component, which are specific to each variable. The relevance of the method is that the DFM is able to extract the few factors that explain the comovement of all South African macroeconomic variables. Forni et al. (2005) demonstrated that when the number of factors is small relative to the number of variables and the panel is heterogeneous, the factors can be recovered from the present and past observations.

Consider an $n \times 1$ covariance stationary process $Y_{t}=\left(y_{1}, \ldots, y_{n}\right)^{\prime}$. Suppose that $X_{t}$ is the standardized version of $Y_{t}$, i.e., $X_{t}$ has a mean zero and a variance equal to one. Under DFM proposed by Forni et al. (2005) $X_{t}$ is described by a factor model, it can be written as the sum of two orthogonal components:

$x_{i t}=b_{i}(L) f_{t}+\xi_{i t}=\lambda_{i} F_{t}+\xi_{i t}$

or, in vector notation:

$X_{t}=B(L) f_{t}+\xi_{i t}=\Lambda F_{t}+\xi_{i t}$

where $f_{t}$ is a $q \times 1$ vector of dynamic factors, $B(L)=B_{0}+B_{1} L+\ldots+B_{s} L^{s}$ is in an $n \times q$ matrix of factor loadings of order $s, \xi_{t}$ is the $n \times 1$ vector of idiosyncratic components, $F_{t}$ is $r \times 1$ vector of static factors, with $r \geqslant q(s+1)$. Let $f_{t}$ and $\xi_{t}$ be mutually orthogonal stationary processes and $\chi_{t}=B(L) f_{t}$ the common component. In factor analysis jargon $X_{t}=B(L) f_{t}+\xi_{i t}$ is referred to as the dynamic factor model, and $X_{t}=\Lambda F_{t}+\xi_{i t}$ as the static factor model. Similarly, $f_{t}$ is regarded as vector of the dynamic factors while $F_{t}$ as the vector of the static factors. Since dynamic common factors are latent, they need to be estimated. Forni et al. (2005) estimate dynamic factors through the use of dynamic principal component analysis. It involves the estimating the eigen values and eigen vectors decomposition of spectral density matrix of $X_{t}$, which is a generalization of orthogonalization process in case of static principal components. The DFM of Forni et al. (2005) is estimated in two steps to solve the end-of-sample problems caused by two-sided filtering encounter with the Dynamic Principle Component Analysis (DPCA) used in Forni et al. (2000). Due to end-of-sample problems this method is not suited for forecasting. Firstly, the DPCA is used to compute estimates of covariance matrices of common and idiosyncratic components of $X_{t}$ at all leads and lags as inverse Fourier transforms of the corresponding estimated spectral density matrices. The spectral density matrix of $X_{t}$ is given by $\Sigma(\theta)=\Sigma_{\chi}(\theta)+\Sigma_{\xi}(\theta)$. Secondly, these estimates are used in the construction of $r$ linear combinations of the observations having smallest idiosyncratic-common variance ratio.

\section{Data}

While, the small-scale, univariate and 3-variable multivariate, VARs, both the classical and Bayesian variants, include data on only the three variables of interest, namely, the annualized real house price growth rates of the large, medium- and small-middle-segment houses, the largescale BVARs and the DFM is estimated based on 268 quarterly series of South Africa, with the data covering the real, nominal and financial sectors. We also have intangible variables, such as confidence indices, and survey variables. In addition to national variables, the paper uses a set of global variables such as commodity industrial inputs price index and crude oil prices. The data also comprises series of major trading partners such as Germany, the United Kingdom (UK), and the United States (US) of America. The in-sample period contains data from 1980:01 to 2000:04, while the out-of-sample set is 2001:012006:04. ${ }^{11}$ All series are seasonally adjusted and were made covariance stationary when estimating the DFM. The more powerful DFGLS test of Elliott et al. (1996), instead of the most popular ADF test, is used to assess the degree of integration of all series. All non-stationary series are made stationary through differencing. The Schwarz information criterion is used in the selecting the appropriate lag length in such a way that no serial correction is left in the stochastic error term. Where there were doubts about the presence of unit root, the KPSS test proposed by Kwiatowski et al. (1992), with the null hypothesis of stationarity, was applied. All series are standardized to have a mean of zero and a constant variance. It must, however, be pointed out that, nonstationarity is not an issue with the BVAR, since Sims et al. (1990) indicate that with the Bayesian approach entirely based on the likelihood function, the associated inference does not need to take special account of nonstationarity, since the likelihood function has the same Gaussian shape regardless of the presence of nonstationarity. Hence, for the sake of comparison amongst the VARs, both classical and Bayesian, we make no attempt to make the variables stationary, unlike in the DFM. ${ }^{12}$

\footnotetext{
${ }^{11}$ Details about data and the statistical treatment of the variables used to estimate the large-scale BVARs and the DFM are available upon request.

12 See Dua and Ray (1995) for further details.
} 
There are various statistical approaches in determining the number of factors in the DFM. For example, Bai and $\mathrm{Ng}$ (2002) developed some criteria guiding the selection of the number of factors in large dimensional panels. The Principal Component Analysis (PCA) can also be used in establishing the number of factors in the DFM. The PCA suggests that the selection of a number of factors $q$ be based on the first eigen values of the spectral density matrix of $X_{t}$. Then, the principal components are added until the increase in the explained variance is less than a specific $\alpha=0.05$. The Bai and $\mathrm{Ng}$ (2002) approach proposes five static factors, while Bai and $\mathrm{Ng}$ (2007) suggests two primitive or dynamic factors. Similar to the latter method, the principal component technique, as proposed by Forni et al. (2000) suggests two dynamic factors. The first two dynamic principal components explain approximately $99 \%$ of variation, while the eigen value of the third component is $0.005(<0.05)$.

\section{Forecasting evaluation}

Given the specifications of the models, we estimate the five alternative types of models, namely, the univariate and multivariate versions of the classical VAR and the smallscale BVARs, the large-scale BVAR and the DFM over the period of 1980:01-2000:04, based on quarterly data. Then we compute the out-of-sample one- through four-quarters-ahead forecasts for the period of 2001:01-2006:04, and compare the forecast accuracy of the alternative models. The different types of the VARs are estimated with eight lags ${ }^{13}$ of each variable. Since we use eight lags, the initial eight quarters of the sample, 1980:01-1981:04, are used to feed the lags. We generate dynamic forecasts, as would naturally be achieved in actual forecasting practice. The models are re-estimated each quarter over the out-of-sample forecast horizon in order to update the estimate of the coefficients, before producing the four-quarters-ahead forecasts. This iterative estimation and four-steps-ahead forecast procedure was carried out for 24 quarters, with the first forecast beginning in 2001:01. This experiment produced a total of 24 one-quarter-ahead forecasts, 24 twoquarters-ahead forecasts, and so on, up to 24 four-stepahead forecasts. The RMSEs ${ }^{14}$ for the 24 , quarter 1 through quarter 4 forecasts are then calculated for the annualized real house price growth rates of the large-, medium- and small-middle-segment housing. The values of the RMSE statistic for one- to four-quarters-ahead forecasts for the period 2001:01-2006:04 are then examined. The model that produces the lowest average value for the RMSE is selected as the 'optimal' model for a specific category of real house price growth rate.

\footnotetext{
13 The choice of eight lags is based on the unanimity of the sequential modified LR test statistic, Akaike Information Criterion (AIC), the Final Prediction Error (FPE) criterion and the Hannan-Quinn (HQ) information criterion applied to a stable VAR estimated with the three variables of concern. Note, stability, as usual, implies that no roots were found to lie outside the unit circle.

${ }^{14}$ Note that if $A_{t+n}$ denotes the actual value of a specific variable in period $t+n$ and ${ }_{t} F_{t+n}$ equals the forecast made in period $t$ for $t+n$, the RMSE statistic equals the following: $\sqrt{\left[\sum_{1}^{N}\left({ }_{t} F_{t+n}-A_{t+n}\right)^{2} / N\right]}$ where $N$ equals the number of forecasts.
}

In Tables 1-3, we compare the RMSEs of one- to fourquarters-ahead out-of-sample-forecasts for the period of 2000:01-2006:04, generated by the abovementioned alternative models. At this stage, a few words need to be said regarding the choice of the evaluation criterion for the out-of-sample forecasts generated from Bayesian models. As Zellner (1986: 494) points out, the 'optimal' Bayesian forecasts will differ depending upon the loss function employed and the form of predictive probability density function. In other words, Bayesian forecasts are sensitive to the choice of the measure used to evaluate the out-ofsample forecast errors. However, Zellner (1986) points out that the use of the mean of the predictive probability density function for a series, is optimal relative to a squared error loss function and the Mean Squared Error (MSE), and hence, the RMSE is an appropriate measure to evaluate performance of forecasts, when the mean of the predictive probability density function is used. This is exactly what we do below in Tables $1-3$, when we use the average RMSEs over the one- to four-quarters-ahead forecasting horizon. ${ }^{15}$ The conclusions, regarding each of the three categories of real house price growth rate, based on the average one- to four-quarters-ahead RMSEs, from these tables can be summarized as follows:

(i) Irrespective of the hyperparameters specifying the tightness of the prior and the size of the houses within the middle-segment category, the large-scale BVARs, outperform all the other models by quite a distance for each of the one- to four-quarters-ahead out-of-sample forecasts. However, within the largescale BVARs, the model with the most tight priors $(w=0.1, d=2.0)$ is the best performing model on average.

(ii) Based on the average RMSEs, the univariate BVAR models with $(w=0.1, d=2.0)$ are a distant second to the large-scale BVARs in each of the three categories of the middle-segment housing. These models are closely followed in the heels by the small-scale BVAR models with same set of hyperparameters specifying the Minnesota prior.

(iii) In all the three cases, the univariate OLS or the Autoregressive model of order 8 , the DFM and the VAR, based on the average RMSEs for the out-of-sample horizon of 2001:01-2006:04, are ranked as fourth, fifth and sixth, respectively.

Note, unlike Das et al. (2008), who found the DFM to be the overwhelming favorite in forecasting regional house price inflation relative to small-scale spatial and non-spatial BVARs and a classical VAR, our results do not indicate so. ${ }^{16}$ In fact, the DFM is found to be ranked below the univariate and multivariate small-scale BVARs in our case.

\footnotetext{
15 Our conclusions were, however, qualitatively the same based on the Mean Absolute Error (MAE) and Mean Absolute Percentage Error (MAPE). The results are available upon request.

16 Interestingly, when we repeated the forecasting exercise for the regional house price inflation using the large-scale BVAR, we found the model to outperform all the "optimal" models of Das et al. (2008). These results are available upon request.
} 
Table 1

RMSEs of one- to four-quarters-ahead (large middle-segment, 2001:01-2006:4).

\begin{tabular}{|c|c|c|c|c|c|c|}
\hline & $\mathrm{QA}$ & 1 & 2 & 3 & 4 & Average \\
\hline & UOLS & 7.6251 & 11.1535 & 12.4930 & 14.6719 & 11.4859 \\
\hline & VAR & 8.2894 & 13.2600 & 16.3651 & 21.7194 & 14.9085 \\
\hline & DFM & 10.7001 & 11.8895 & 12.3906 & 13.6091 & 12.1473 \\
\hline \multirow[t]{3}{*}{$w=0.3, d=0.5$} & UBVAR & 7.6507 & 10.8855 & 12.3250 & 14.0990 & 11.2400 \\
\hline & SBVAR & 7.1261 & 10.0614 & 11.5019 & 13.6714 & 10.5902 \\
\hline & LBVAR & 2.5138 & 0.8128 & 1.7729 & 0.9020 & 1.5003 \\
\hline \multirow[t]{3}{*}{$w=0.2, d=1$} & UBVAR & 7.6156 & 10.2381 & 11.5509 & 13.1236 & 10.6320 \\
\hline & SBVAR & 7.8267 & 10.7540 & 12.1414 & 13.6428 & 11.0912 \\
\hline & LBVAR & 2.3499 & 0.3116 & 2.1669 & 1.0100 & 1.4596 \\
\hline \multirow[t]{3}{*}{$w=0.1, d=1$} & UBVAR & 7.7395 & 10.3749 & 11.4148 & 12.8624 & 10.5979 \\
\hline & SBVAR & 7.7199 & 10.3497 & 11.3774 & 12.8060 & 10.5633 \\
\hline & LBVAR & 2.5542 & 0.4357 & 1.7550 & 0.7009 & 1.3614 \\
\hline \multirow[t]{3}{*}{$w=0.2, d=2$} & UBVAR & 7.9524 & 10.8216 & 12.2585 & 13.7656 & 11.1995 \\
\hline & SBVAR & 7.8224 & 10.3813 & 11.6917 & 13.1564 & 10.7630 \\
\hline & LBVAR & 1.7410 & 0.2447 & 2.2942 & 1.0257 & 1.3264 \\
\hline \multirow[t]{3}{*}{$w=0.1, d=2$} & UBVAR & 7.7368 & 10.2601 & 11.2240 & 12.6499 & 10.4677 \\
\hline & SBVAR & 7.7718 & 10.3328 & 11.3300 & 12.7285 & 10.5408 \\
\hline & LBVAR & 2.1479 & 0.2178 & 1.7364 & 0.5203 & 1.1556 \\
\hline
\end{tabular}

UBVAR, Univariate BVAR; SBVAR, Small-Scale BVAR; LBVAR, Large-Scale BVAR.

Table 2

RMSEs of one- to four-quarters-ahead (medium middle-segment, 2001:01-2006:4).

\begin{tabular}{|c|c|c|c|c|c|c|}
\hline & QA & 1 & 2 & 3 & 4 & Average \\
\hline & UOLS & 6.6699 & 11.1283 & 13.7053 & 15.9012 & 11.8512 \\
\hline & VAR & 7.6226 & 13.2491 & 17.7929 & 22.7807 & 15.3613 \\
\hline & DFM & 11.0542 & 12.3504 & 13.1730 & 14.5277 & 12.7764 \\
\hline \multirow[t]{3}{*}{$w=0.3, d=0.5$} & UBVAR & 6.6217 & 10.8245 & 13.5545 & 15.5817 & 11.6456 \\
\hline & SBVAR & 6.5071 & 10.5573 & 13.2145 & 15.4633 & 11.4355 \\
\hline & LBVAR & 1.3811 & 1.7065 & 0.3441 & 1.2291 & 1.1652 \\
\hline \multirow[t]{3}{*}{$w=0.2, d=1$} & UBVAR & 6.5061 & 10.4036 & 13.0992 & 15.1067 & 11.2789 \\
\hline & SBVAR & 6.5437 & 10.4656 & 13.2209 & 15.1911 & 11.3553 \\
\hline & LBBAR & 1.345286 & 1.668599 & 0.215229 & 1.421703 & 1.1627 \\
\hline \multirow[t]{3}{*}{$w=0.1, d=1$} & UBVAR & 6.2777 & 9.8641 & 12.4356 & 14.3960 & 10.7434 \\
\hline & SBVAR & 6.2935 & 9.9233 & 12.5298 & 14.5264 & 10.8183 \\
\hline & LBVAR & 1.228055 & 1.45676 & 0.252001 & 1.29891 & 1.0589 \\
\hline \multirow[t]{3}{*}{$w=0.2, d=2$} & UBVAR & 6.4954 & 10.2068 & 12.8802 & 14.8433 & 11.1064 \\
\hline & SBVAR & 6.4512 & 10.1520 & 12.7567 & 14.7452 & 11.0263 \\
\hline & LBVAR & 0.808409 & 1.32817 & 0.125094 & 1.331778 & 0.8984 \\
\hline \multirow[t]{3}{*}{$w=0.1, d=2$} & UBVAR & 6.2212 & 9.6563 & 12.1248 & 14.0596 & 10.5155 \\
\hline & SBVAR & 6.2391 & 9.7022 & 12.2007 & 14.1637 & 10.5764 \\
\hline & LBVAR & 0.731027 & 1.00139 & 0.002744 & 0.933318 & 0.6671 \\
\hline
\end{tabular}

UBVAR, Univariate BVAR; SBVAR, Small-Scale BVAR; LBVAR, Large-Scale BVAR.

However, there is no doubt over the capability of the largescale BVARs in forecasting the economy-wide annualized real house price growth rates of the large-, medium- and small-middle-segment housing.

At this stage, we must, however, point to at least two limitations of the Bayesian approach: First, as is clear from Tables $1-3$, the forecast accuracy is sensitive to the choice of the priors. So if the prior is not well specified, an alternative model used for forecasting may perform better. Second, for the Bayesian models, one requires to specify an objective function, for example the average RMSEs, to search for the 'optimal' priors, which in turn, needs to be optimized over the period for which we compute the out-of-sample forecasts. However, there is no guarantee that the chosen parameter values specifying the prior will continue to be 'optimal' beyond the period for which it was selected. Nevertheless, the role of BVARs in forecasting macroeconomic variables accurately cannot be underestimated.

\section{Predicting the turning points}

In what follows, we look at the ability of the large-scale BVAR model with $(w=0.1, d=2.0)$ in predicting the recent downturn in the real house price growth rate over 2003:01-2008:02, in comparison to the AR(8), the VAR, the DFM and those BVARs, univariate and multivariate, that on average produces the minimum average RMSEs 
Table 3

RMSEs of one- to four-quarters-ahead (small middle-segment, 2001:01-2006:4)

\begin{tabular}{|c|c|c|c|c|c|c|}
\hline & $\mathrm{QA}$ & 1 & 2 & 3 & 4 & Average \\
\hline & UOLS & 7.1218 & 10.9471 & 13.3339 & 16.0891 & 11.8730 \\
\hline & VAR & 7.9998 & 13.5224 & 18.1246 & 23.6199 & 15.8167 \\
\hline & DFM & 11.3963 & 11.6307 & 12.1631 & 14.2256 & 12.3539 \\
\hline \multirow[t]{3}{*}{$w=0.3, d=0.5$} & UBVAR & 7.0812 & 10.9023 & 13.4321 & 15.8916 & 11.8268 \\
\hline & SBVAR & 6.7764 & 10.6527 & 13.4899 & 16.2816 & 11.8001 \\
\hline & LBVAR & 2.2690 & 5.2247 & 3.5924 & 3.7260 & 3.7030 \\
\hline \multirow[t]{3}{*}{$w=0.2, d=1$} & UBVAR & 7.0021 & 10.7143 & 13.2513 & 15.5501 & 11.6295 \\
\hline & SBVAR & 7.1894 & 10.9627 & 13.4611 & 15.6902 & 11.8259 \\
\hline & LBVAR & 1.6219 & 3.9732 & 2.3725 & 2.8725 & 2.7100 \\
\hline \multirow[t]{3}{*}{$w=0.1, d=1$} & UBVAR & 7.1012 & 10.6804 & 12.9729 & 15.2257 & 11.4951 \\
\hline & SBVAR & 7.0560 & 10.6301 & 12.9526 & 15.2829 & 11.4804 \\
\hline & LBVAR & 1.6947 & 3.8056 & 2.6036 & 2.8698 & 2.7434 \\
\hline \multirow[t]{3}{*}{$w=0.2, d=2$} & UBVAR & 7.2474 & 10.9464 & 13.3839 & 15.5598 & 11.7844 \\
\hline & SBVAR & 7.0765 & 10.6763 & 13.1243 & 15.3775 & 11.5637 \\
\hline & LBVAR & 1.0821 & 3.0972 & 2.1133 & 3.0300 & 2.3307 \\
\hline \multirow[t]{3}{*}{$w=0.1, d=2$} & UBVAR & 7.0911 & 10.5807 & 12.8007 & 15.0435 & 11.3790 \\
\hline & SBVAR & 7.0804 & 10.5700 & 12.8116 & 15.1174 & 11.3948 \\
\hline & LBVAR & 1.2918 & 3.1277 & 2.1990 & 2.6840 & 2.3256 \\
\hline
\end{tabular}

UBVAR, Univariate BVAR; SBVAR, Small-Scale BVAR; LBVAR, Large-Scale BVAR.

for specific values of $w$ and $d$, specifying the Minnesota prior. As with the large-scale BVAR, the univariate and multivariate small-scale BVARs that outperforms the other models within its own category also has a value of $w=0.1$, $d=2.0$. The decision to choose 2003:01 as the starting date for the models to predict the turning points is simply because the real house price growth rates in the middle-segment of the housing peaked at 2003:04 as depicted in Fig. 1. Given that ideally one would want to use available information prior to the turning point, following the methodology of Gupta and Das (forthcoming), all the 'optimal' models are estimated till 2002:04 and then we forecast over the period of 2003:01 till 2008:02.

As can be observed from Figs. 2-4, the 'optimal' largescale BVAR with $(w=0.1, d=2.0)$ is clearly the best suited model in predicting the recent downturn in the real house price growth rates of the large-, medium- and small-middle-segment housing over the period of 2003:012008:02. In general, the optimal large-scale BVAR tends to underpredict over the period of 2003:01 till 2006:04 and over predict the downturn especially when the real growth rate becomes negative in case of the large- and

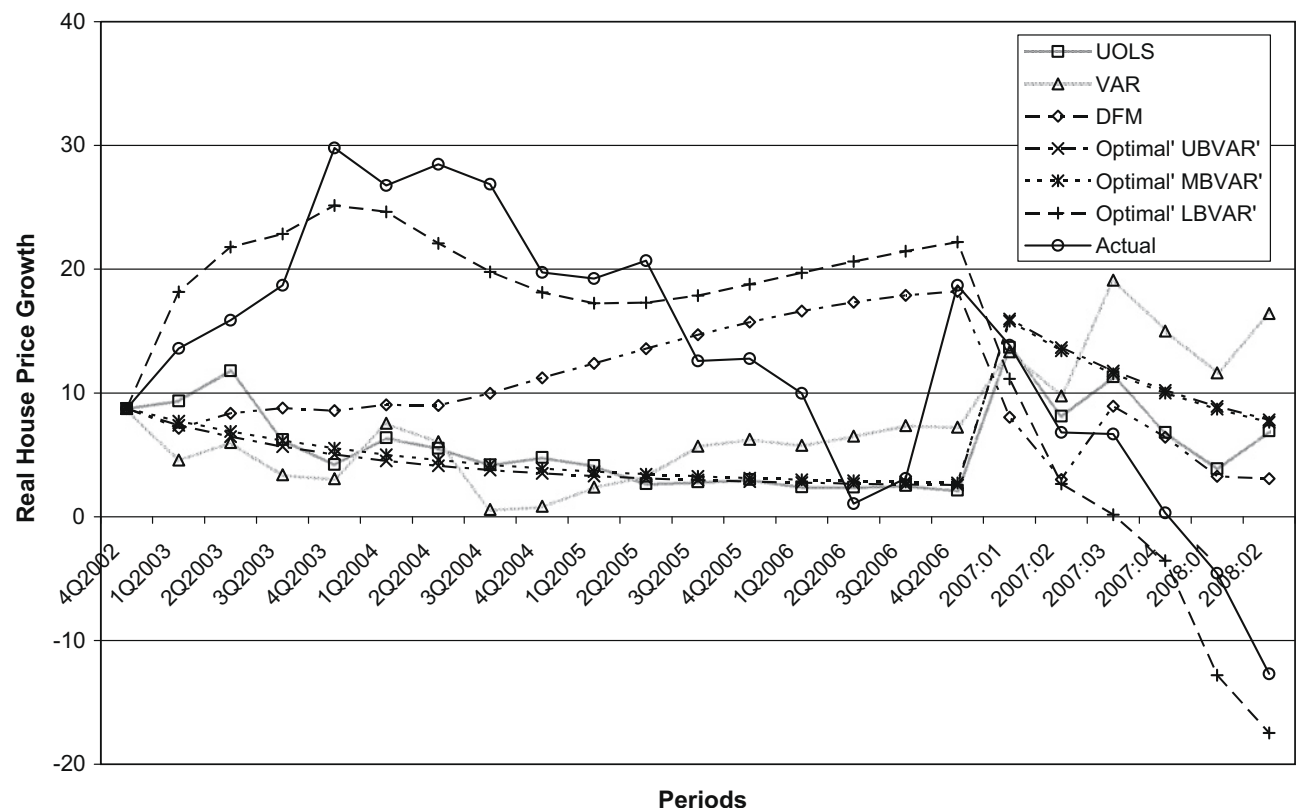

Fig. 2. Predicting the turning points in real house price growth (large middle-segment, 2003Q1-2008Q2). 


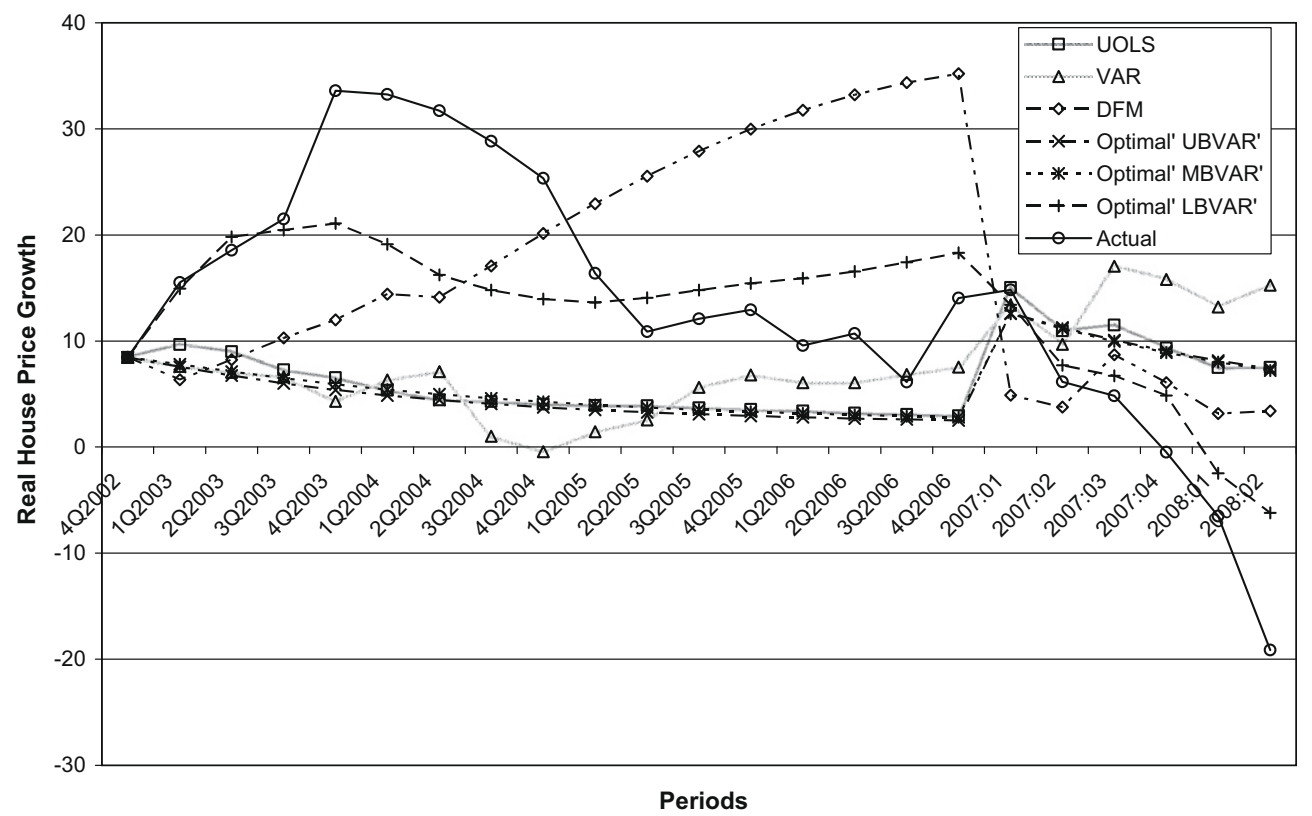

Fig. 3. Predicting the turning points in real house price growth (medium middle-segment, 2003Q1-2008Q4).

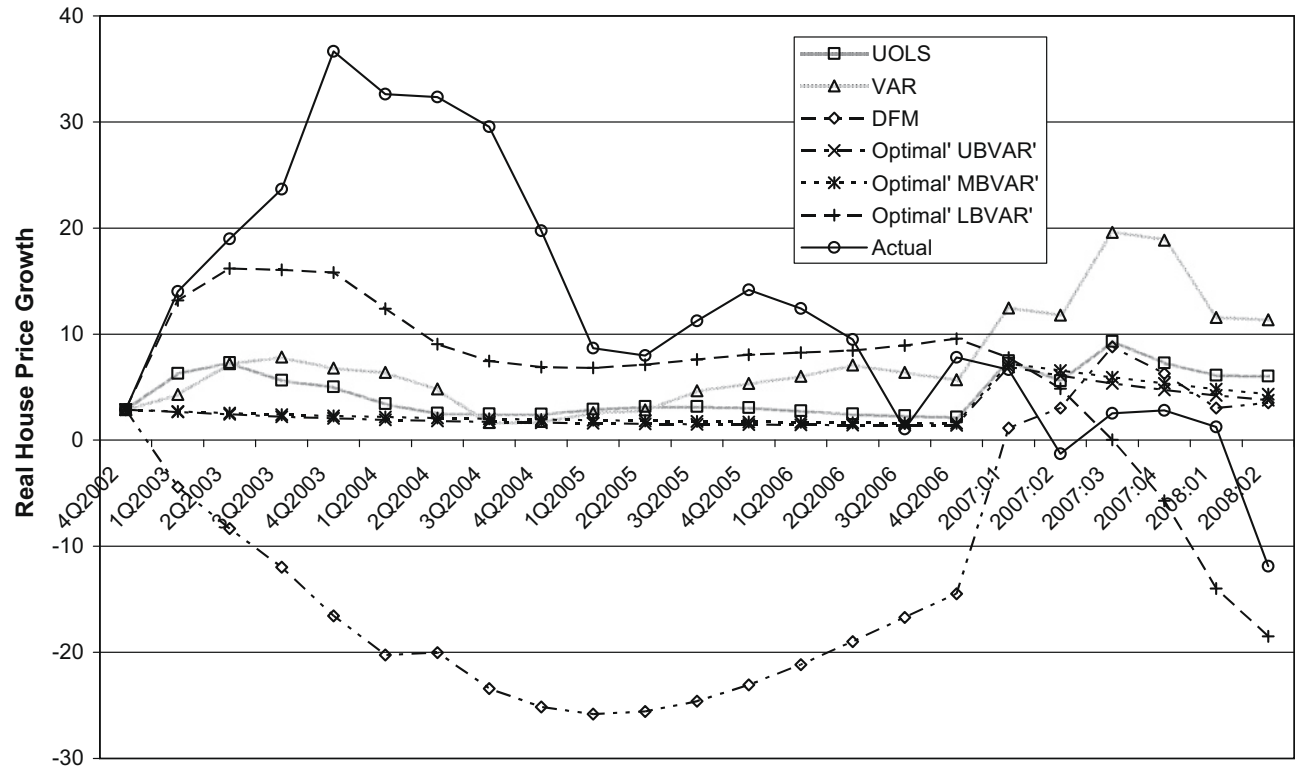

Periods

Fig. 4. Predicting the turning points in real house price growth (small middle-segment, 2003Q1-2008Q2).

small-middle-segment housing in the latter half of the horizon. The fact that the 'optimal' large-scale BVAR does so well in predicting the recent downturn relative to the 'optimal' small-scale univariate and multivariate BVARs, is clearly an indication of the role fundamentals play in affecting real house prices, over and beyond the information contained in the growth rates of the lagged real house prices. Moreover, given that the large-scale BVAR model allows for the domestic variables to have a minimal effect on foreign and world variables, while the latter set of vari- ables to have a strong influence on the South African variables, might be causing it to perform better than the DFM, which also includes the same set of variables, both in terms of forecasting and predicting the recent downturn.

\section{Conclusions}

This paper develops large-scale BVAR models, based on 268 quarterly series, for forecasting annualized real house 
price growth rates for large-, medium- and small-middlesegment housing for the South African economy. Given the in-sample period of 1980:01-2000:04, the large-scale BVARs, estimated under alternative hyperparameter values specifying the priors, are used to forecast real house price growth rates over a 24-quarter out-of-sample horizon of 2001:01-2006:04. The forecast performance of the largescale BVARs are then compared with classical and Bayesian versions of univariate and multivariate VAR models, comprising of the real growth rates of the large-, mediumand small-middle-segment houses only, and a large-scale DFM, which comprises of the same 268 variables included in the large-scale BVARs. Once we determine a model that produces, on average, the minimum one- to four-quartersahead RMSEs over the out-of-sample horizon, we evaluate how well the same would have been able to predict the recent downturn in the real house price growth rates for the three categories of the middle-segment-housing over the period of 2003:01-2008:02.

Our results indicate that, irrespective of the hyperparameters specifying the tightness of the prior and the size of the houses within the middle-segment category, the large-scale BVARs outperform all the other models by a distance for each of the one- to four-quarters-ahead out-ofsample forecasts. However, within the large-scale BVARs, the model with the most tight priors $(w=0.1, d=2.0)$ is the best performing model on average. Moreover, this 'optimal' large-scale BVAR is also capable of tracking closely the recent downturn in the real house price growth rates for the three categories of the middle-segment-housing over an ex ante period of 2003:01-2008:02. In summary, we find a tight-priored large-scale BVAR model, which not only includes the widest possible set of fundamentals that tends to affect the housing market, but also treats South Africa as a small open economy by allowing for asymmetry in the specification of the prior, is the overwhelming favorite to forecast and predict turning points for the middle-segment category of housing. At this juncture, we must elaborate that given the type of models that we use, the study can easily be carried out for any country(ies).

There are, however, as noted earlier, limitations to using the BVAR approach. First, the forecast accuracy depends critically on the specification of the prior, and second, the selection of the prior based on some objective function for the out-of-sample forecasts may not be 'optimal' for the time period beyond the period chosen to produce the out-of-sample forecasts. Besides these, there are two other major concerns, which are, however general, to the traditional statistically estimated models used, like the VARs both Classical and Bayesian and the DFM, for forecasting at the business cycle frequencies. First, such procedures perform reasonably well as long there are no structural changes experienced by the economy. Such changes, whether in or out of the sample, would then entail the models inappropriate. Alternatively, these models are not immune to the 'Lucas Critique'. ${ }^{17}$ Furthermore, the estimation procedures used here are linear in nature, and hence, they fail to take into account of the nonlinearities in the data. One and, perhaps, the best response to these objections has been the development of micro-founded DSGE models, which are capable of handling both the problems arising out of the structural changes and the issues of nonlinearities. ${ }^{18}$ As in Iacoviello and Neri (2008), future research should concentrate on using DSGE models to model the housing sector of an economy, and then using the same to forecast house prices.

\section{References}

Abraham, J.M., Hendershott, P.H., 1996. Bubbles in metropolitan housing markets. Journal of Housing Research 7, 191-207.

ABSA Housing Price Review, 2008. Quarter 1, Quarter 2 and Quarter 3.

Bai, J., Ng, S., 2007. Determining the number of primitive shocks in factor models. Journal of Business and Economic Statistics 25, 52-60.

Bai, J., Ng, S., 2002. Determining the number of factors in approximate factor models. Econometrica 70, 191-221.

Banerji, A., Dua, P., Miller, S.M., 2008. Performance evaluation of the new connecticut leading employment index using lead profiles and BVAR models. Journal of Forecasting 25, 415-437

Bernanke, B., Gertler, M., 1995. Inside the black box: the credit channel of monetary transmission. Journal of Economic Perspectives 9, 27-48.

Burger, P., Van Rensburg, L.J., 2008. Metropolitan house prices in South Africa: Do they converge? South African Journal of Economics 76, 291-297.

Cho, M., 1996. House price dynamics: a survey of theoretical and empirical issues. Journal of Housing Research 7, 145-172.

Das, S., Gupta, R., Kabundi, A., 2008. Is a DFM well-suited for forecasting regional house price inflation? Economic Research Southern Africa Working Paper No. 85.

Del Negro, M., 2001. Turn, turn, turn: predicting turning points in economic activity. Economic Review. Second Quarter, Federal Reserve Bank of Atlanta.

Doan, T.A., Litterman, R.B., Sims, C.A., 1984. Forecasting and conditional projections using realistic prior distributions. Econometric Reviews 3, $1-100$.

Dua, P., Smyth, D.J., 1995. Forecasting U.S. home sales using BVAR models and survey data on households' buying attitude for homes. Journal of Forecasting 14, 217-227.

Dua, P., Miller, S.M., Smyth, D.J., 1999. Using leading indicators to forecast U.S. home sales in a bayesian vector autoregressive framework. Journal of Real Estate Finance and Economics 18, 191-205.

Dua, P., Miller, S.M., 1996. Forecasting connecticut home sales in a BVAR framework using coincident and leading indexes. Journal of Real Estate Finance and Economics 13, 219-235

Dua, P., Ray, S.C., 1995. A BVAR model for the connecticut economy. Journal of Forecasting 14, 167-180.

Elliott, G., Rothenberg, T.J., Stock, J., 1996. Efficient tests for an autoregressive unit root. Econometrica 64, 813-836.

Forni, M., Hallin, M., Lippi, M., Reichlin, L., 2005. The generalized dynamic factor model, one sided estimation and forecasting. Journal of the American Statistical Association 100, 830-840.

Forni, M., Hallin, M., Lippi, M., Reichlin, L., 2003. Do financial variables help forecasting inflation and real activity in the euro area? Journal of Monetary Economics 50, 1243-1255.

Forni, M., Hallin, M., Lippi, M., Reichlin, L., 2000. The generalized dynamic factor model: identification and estimation. Review of Economics and Statistics 82, 540-554.

Gupta, R., 2009. Bayesian methods of forecasting inventory investment. South African Journal of Economics 77, 113-126.

Gupta, R., Das, S., 2008. Spatial Bayesian methods for forecasting house prices in six metropolitan areas of South Africa. South African Journal of Economics 76, 298-313.

Gupta, R., Das, S., forthcoming. Predicting Downturns in the US Housing Market. Journal of Real Estate Economics and Finance.

Gupta, R., Kabundi, A., 2008a. A dynamic factor model for forecasting macroeconomic variables in South Africa. Working Paper No. 200815. Department of Economics, University of Pretoria.

Gupta, R., Kabundi, A., 2008b. Forecasting macroeconomic variables using large datasets: dynamic factor model vs large-scale BVARs. Working Paper No. 200816. Department of Economics, University of Pretoria.

\footnotetext{
17 See Lucas (1976) for details.
} 
Gupta, R., Kabundi, A., 2008c. Forecasting macroeconomic variables in a small open economy: a comparison between small- and large-scale models. Working Paper No. 200830. Department of Economics, University of Pretoria.

Gupta, R., 2006. Forecasting the South African economy with VARs and VECMs. South African Journal of Economics 74, 611-628.

Gupta, R., Sichei, M., 2006. A BVAR model for the South African economy. South African Journal of Economics 74, 391-409.

Iacoviello, M., Neri, S., 2008. Housing market spillovers: evidence from an estimated DSGE model. Paper provided by Boston College Department of Economics in its series Boston College. Working Papers in Economics with Number 659.

International Monetary Fund, 2000. World Economic Outlook: Asset Prices and the Business Cycle.

Johnes, G., Hyclak, T., 1999. House prices and regional labor markets. Annals of Regional Science 33, 33-49.

Kinal, T., Ratner, J., 1986. A VAR forecasting model of a regional economy: its construction and comparison. International Regional Science Review 10, 113-126.

Kwiatowski, D., Phillips, P.C.B., Schmidt, P., Shin, Y., 1992. Testing the null hypothesis of stationarity against the alternative of a unit root: How sure are we that economic time series have a unit root? Journal of Econometrics 54, 159-178.

Litterman, R.B., 1981. A Bayesian procedure for forecasting with vector autoregressions. Working Paper. Federal Reserve Bank of Minneapolis.

Litterman, R.B., 1986. Forecasting with Bayesian vector autoregressions five years of experience. Journal of Business and Economic Statistics 4, 25-38.

Liu, G., Gupta, R., 2007. A small-scale DSGE model for forecasting the South African economy. South African Journal of Economics 75, 179193.

Liu, G., Gupta, R., Schaling, E., forthcoming-a. Forecasting the South African economy: a hybrid-DSGE approach. Journal of Economic Studies.
Liu, G., Gupta, R., Schaling, E., forthcoming-b. A New Keynesian DSGE model for forecasting the South African economy. Journal of Forecasting.

Lucas Jr., R.E., 1976. Econometric policy evaluation: a critique. Carnegie Rochester Conference Series on Public Policy 1, 19-46.

Rapach, D.E., Strauss, J.K., 2009. Differences in Housing Price Forecastability across U.S. States. International Journal of Forecasting 25, 351-371.

Rapach, D.E., Strauss, J.K., 2007. Forecasting real housing price growth in the eighth district states. Federal Reserve Bank of St. Louis. Regional Economic Development 3, 33-42.

Shoesmith, G.L., 1992. Co-integration, error correction and medium-term regional VAR forecasting. Journal of Forecasting 11, 91-109.

Sims, C.A., Stock, J.H., Watson, M.W., 1990. Inference in linear time series models with some unit roots. Econometrica 58, 113-144.

Sims, C.A., 1980. Macroeconomics and reality. Econometrica 48, 1-48.

Spencer, D.E., 1993. Developing a Bayesian vector autoregression model. International Journal of Forecasting 9, 407-421.

Stock, J.H., Watson, M.W., 2003. Forecasting output and inflation: the role of asset prices. Journal of Economic Literature 41, 788-829.

Theil, H., 1971. Principles of Econometrics. John Wiley, New York.

Todd, R.M., 1984. Improving economic forecasting with Bayesian vector autoregression. Quarterly Review Federal Reserve Bank of Minneapolis Fall, 18-29.

Topel, R.H., Rosen, S., 1988. Housing investment in the United States. Journal of Political Economy 96, 718-740.

Vargas-Silva, C., 2008. The effect of monetary policy on housing: a factor augmented vector autoregression (FAVAR) approach. Applied Economics Letters 15, 749-752.

Zellner, A., 1986. A tale of forecasting 1001 series: the Bayesian knight strikes again. International Journal of Forecasting 2, 494.

Zita, S.E., Gupta, R., 2008. Modelling and forecasting the metical-rand exchange rate. ICFAI Journal of Monetary Economics VI, 63-90. 